\title{
Oscillation Results for a Class of Fourth-Order Nonlinear Differential Equations with Positive and Negative Coefficients
}

\author{
Pakize Temtek and Nagehan Kılınç Geçer*
}

(Communicated by İ. Onur KIYMAZ)

\begin{abstract}
We are interested in oscillation of the fourth-order nonlinear differential equations of the form$$
\left(r_{1}(t)(x(t)+p(t) x(\sigma(t)))^{\prime \prime}\right)^{\prime \prime}+\sum_{i=1}^{\ell} q_{i}(t) G\left(x\left(\tau_{i}(t)\right)\right)-\sum_{i=1}^{\ell} h_{i}(t) H\left(x\left(\rho_{i}(t)\right)\right)=0
$$

under the assumption that

$$
\int_{0}^{\infty} \frac{t}{r_{1}(t)} d t<\infty
$$

for different ranges of $p(t)$.

Keywords: Oscillation; fourth order nonlinear differential equation.

AMS Subject Classification (2010): 34C10; 35C15.

${ }^{*}$ Corresponding author
\end{abstract}

\section{Introduction}

Over the past few years, there has been a strong concern in the study of the oscillatory behavior of solutions of delay differential equations with positive and negative coefficients of the first and second orders; see, e.g., [1, 5-8, 10-11]. In this paper, we consider the nonlinear fourth-order delay differential equations of the form

$$
\left(r_{1}(t)(x(t)+p(t) x(\sigma(t)))^{\prime \prime}\right)^{\prime \prime}+\sum_{i=1}^{\ell} q_{i}(t) G\left(x\left(\tau_{i}(t)\right)\right)-\sum_{i=1}^{\ell} h_{i}(t) H\left(x\left(\rho_{i}(t)\right)\right)=0
$$

where $r_{1}, \sigma, q_{i}, \tau_{i}, h_{i}, \rho_{i}$ are continuous and positive on $[0, \infty), i \in\{1,2, \ldots, \ell\} p \in C([0, \infty), \mathbb{R}), G, H \in C(\mathbb{R}, \mathbb{R})$ with $d G(d)>0$ and $b H(b)>0$ for $d, b \neq 0, H$ is bounded, $G$ is nondecreasing. Further $\sigma(t) \leq t, \lim _{t \rightarrow \infty} \sigma(t)=\infty$, and $\sigma_{1}$ is a positive constant such that $\sigma_{1}<\sigma(t) \leq t$. And $\tau \in C([0, \infty), \mathbb{R})$ such that $\tau(t) \leq \tau_{i}(t) \leq t$ for $i \in\{1,2, \ldots, \ell\}$, and $\lim _{t \rightarrow \infty} \tau(t)=\infty$ and $\rho \in C([0, \infty), \mathbb{R})$ such that $\rho(t) \leq \rho_{i}(t) \leq t$ for $i \in\{1,2, \ldots, \ell\}, \lim _{t \rightarrow \infty} \rho(t)=\infty$.

The main object of our work is to investigate the oscillatory and asymptotic behaviors of the solutions of (1.1) 
under the assumption that

$$
\left(H_{1}\right) \quad \int_{0}^{\infty} \frac{t}{r_{1}(t)} d t<\infty .
$$

If $\sigma(t)=t-\tau$ and $\sum_{i=1}^{\ell} q_{i}(t) G\left(x\left(\tau_{i}(t)\right)\right)-\sum_{i=1}^{\ell} h_{i}(t) H\left(x\left(\rho_{i}(t)\right)\right)=q(t) G(x(t-\alpha))$, then (1.1) reduced to

$$
\left(r_{1}(t)(x(t)+p(t) x(t-\tau))^{\prime \prime}\right)^{\prime \prime}+q(t) G(x(t-\alpha))=0
$$

where $G \in C(\mathbb{R}, \mathbb{R})$ with $d G(d)>0, d \neq 0, G$ is nondecreasing, $\tau, \alpha>0$ are constants. In[4], Parhi and Tripathy studied Eq. (1.2) under the assumption $\left(H_{1}\right)$.

Tripathy et al.[2] considered nonlinear fourth-order neutral delay differential equations of the form

$$
\left(r(t)(y(t)+p(t) y(t-\tau))^{\prime \prime}\right)^{\prime \prime}+q(t) G(y(t-\alpha))-h(t) H(y(t-\beta))=0
$$

where $G, H$ has the same properties with us and $\tau, \alpha, \beta>0$ are constants.

They studied (1.3) under the same assumption in addition to

$$
\left(H_{2}\right) \quad \int_{0}^{\infty} \frac{s}{r_{1}(s)} \int_{s}^{\infty} t h(t) d t d s<\infty .
$$

Since Eq. (1.1) is more general than Eqs. (1.2) and (1.3), it is worth studying. Not only the present work is more illustrative than [2] but also some of results are generalized and improved. A solution of (1.1) is understood as a function $x \in C([-\eta, \infty), \mathbb{R})$ such that $(x(t)+p(t) x(\sigma(t)))$ and $\left(r_{1}(t)(x(t)+p(t) x(\sigma(t)))^{\prime \prime}\right)$ are twice continuously differentiable, and (1.1) is satisfied for $t \geq 0$, where $\eta=\max \left\{\sigma, \tau_{i}, \rho_{i}\right\}$,

$$
\sup \left\{x(t): t \geq t_{0}\right\}>0
$$

for every $t \geq t_{0}$. A solution $x(t)$ of (1.1) is called oscillatory if it is neither eventually positive nor eventually negative, and it is called nonoscillatory otherwise.

\section{Preliminaries}

We begin with the following results frequently used in what follows:

Lemma 2.1. [4] Let $\left(H_{1}\right)$ hold. If $f(t)$ is an eventually positive twice continuously differentiable function such that $r_{1}(t) f^{\prime \prime}(t)$ is twice continuously differentiable and

$$
\left(r_{1}(t) f^{\prime \prime}(t)\right)^{\prime \prime} \leq 0, \quad \not \equiv 0
$$

for large $t$, where $r_{1} \in C([0, \infty),(0, \infty))$, then one of the following cases holds for large $t$ :

(a) $f^{\prime}(t)>0, f^{\prime \prime}(t)>0$ and $\left(r_{1}(t) f^{\prime \prime}(t)\right)^{\prime}>0$,

(b) $f^{\prime}(t)>0, f^{\prime \prime}(t)<0$ and $\left(r_{1}(t) f^{\prime \prime}(t)\right)^{\prime}>0$,

(c) $f^{\prime}(t)>0, f^{\prime \prime}(t)<0$ and $\left(r_{1}(t) f^{\prime \prime}(t)\right)^{\prime}<0$,

(d) $f^{\prime}(t)<0, f^{\prime \prime}(t)>0$ and $\left(r_{1}(t) f^{\prime \prime}(t)\right)^{\prime}>0$.

Lemma 2.2. [4] Assume that the conditions of Lemma 2.1 are satisfied. Then

(i) the following inequalities hold for large $t$ in the case (c) of Lemma 2.1

$$
\begin{gathered}
f^{\prime}(t) \geq-\left(r_{1}(t) f^{\prime \prime}(t)\right)^{\prime} R(t), f^{\prime}(t) \geq-r_{1}(t) f^{\prime \prime}(t) \int_{t}^{\infty} \frac{d s}{r_{1}(s)}, \\
f(t) \geq k t f^{\prime}(t) \text { and } f(t) \geq-k\left(r_{1}(t) f^{\prime \prime}(t)\right)^{\prime} t R(t),
\end{gathered}
$$

where $k>0$ is a constant and 


$$
R(t)=\int_{t}^{\infty} \frac{s-t}{r_{1}(s)} d s
$$

and

(ii) $f(t) \geq r_{1}(t) f^{\prime \prime}(t) R(t)$ for large $t$ in the case (d) of Lemma 2.1.

Lemma 2.3. [4] If the conditions of Lemma 2.1 are satisfied, then there exist constants $k_{1}>0$ and $k_{2}>0$ such that $k_{1} R(t) \leq f(t) \leq k_{2}$ for large $t$.

Lemma 2.4. [4] Let $\left(H_{1}\right)$ hold. Suppose that $z(t)$ is a real-valued twice continuously differentiable function on $[0, \infty)$ such that $\left(r_{1}(t) z^{\prime \prime}(t)\right)^{\prime \prime} \leq 0, \quad \not \equiv 0$ for large $t$. If $z(t)>0$ eventually, then one of the following cases holds for large $t$ :

(a) $z^{\prime}(t)>0, z^{\prime \prime}(t)>0$ and $\left(r_{1}(t) z^{\prime \prime}(t)\right)^{\prime}>0$,

(b) $z^{\prime}(t)>0, z^{\prime \prime}(t)<0$ and $\left(r_{1}(t) z^{\prime \prime}(t)\right)^{\prime}>0$,

(c) $z^{\prime}(t)>0, z^{\prime \prime}(t)<0$ and $\left(r_{1}(t) z^{\prime \prime}(t)\right)^{\prime}<0$,

(d) $z^{\prime}(t)<0, z^{\prime \prime}(t)>0$ and $\left(r_{1}(t) z^{\prime \prime}(t)\right)^{\prime}>0$.

If $z(t)<0$ for large $t$, then either one of the cases $(b)-(d)$ holds or one of the following cases holds for large $t$ :

(e) $z^{\prime}(t)<0, z^{\prime \prime}(t)<0$ and $\left(r_{1}(t) z^{\prime \prime}(t)\right)^{\prime}>0$,

(f) $z^{\prime}(t)<0, z^{\prime \prime}(t)<0$ and $\left(r_{1}(t) z^{\prime \prime}(t)\right)^{\prime}<0$.

Lemma 2.5. [3] Let $p, x, z \in C([0, \infty), \mathbb{R})$ be such that

$$
z(t)=x(t)+p(t) x(t-\tau)
$$

for $t \geq \tau \geq 0, x(t)>0$ for $t \geq t_{1}>\tau \liminf _{t \rightarrow \infty} x(t)=0$, and $\lim _{t \rightarrow \infty} z(t)=L$ exists. Also let $p(t)$ satisfy one of the following conditions:

(i) $0 \leq p(t) \leq p_{1}<1$,

(ii) $1<p_{2} \leq p(t) \leq p_{3}$ ，

(iii) $p_{4} \leq p(t) \leq 0$,

where $p_{i}$ is a constant, $1 \leq i \leq 4$. Then $L=0$

\section{Main Results}

In this section, sufficient conditions are established for the unbounded oscillation and asymptotic behavior of the solutions of (1.1) under the assumption $\left(H_{1}\right)$. For our aim, we need the following assumptions:

$\left(H_{3}\right) \lambda>0$ such that $G(d)+G(b) \geq \lambda G(d+b)$ for $d, b>0, d, b \in \mathbb{R}$,

$\left(H_{4}\right) G(d b)=G(d) G(b), d, b \in \mathbb{R}$,

$\left(H_{5}\right) G(-d)=-G(d)$, and $H(-d)=-H(d), d \in \mathbb{R}$,

$\left(H_{6}\right) \int_{\sigma_{1}}^{\infty} Q(t) d t=\infty, Q(t)=\min \{q(t), q(\sigma(t))\}, t \geq \sigma_{1}$,

$\left(H_{7}\right) \int_{t_{0}}^{\infty} b(t) Q(t) \sum_{i=1}^{\ell} G\left(R\left(\tau_{i}(t)\right)\right) d t=\infty$, where $b(t)=\min \left\{R^{\gamma}(t), R^{\gamma}(\sigma(t))\right\}$, 
$\gamma>1, t_{0} \geq \eta>0$,

$\left(H_{8}\right) \int_{t_{0}}^{\infty} R^{\gamma}(t) \sum_{i=1}^{\ell} G\left(R\left(\tau_{i}(t)\right)\right) q_{i}(t) d t=\infty, \gamma>1, t_{0} \geq \eta>0$.

Remark 3.1. Since

$$
R(t)<\int_{t}^{\infty} \frac{s}{r_{1}(s)} d s
$$

we conclude that $R(t) \rightarrow 0$ as $t \rightarrow \infty$ in view of $\left(H_{1}\right)$.

Remark 3.2. Assumption $\left(H_{4}\right)$ implies that $G(-d)=-G(d)$. Indeed,

$G(1) G(1)=G(1)$ and $G(1)>0$ imply that $G(1)=1$. Further, $G(-1) G(-1)=G(1)=1$ implies that $(G(-1))^{2}=1$. Since $G(-1)<0$, we conclude that $G(-1)=-1$. Hence,

$$
G(-d)=G(-1) G(d)=-G(d) .
$$

Moreover, $G(x y)=G(x) G(y)$ for every $x, y \in \mathbb{R}$ such that $G(d b)=G(d) G(b)$ for $d>0$ and $b>0$ and $G(-d)=$ $-G(d)$. In addition, the prototype of $G$ satisfying $\left(H_{3}\right),\left(H_{4}\right)$ and $\left(H_{5}\right)$ is

$$
G(u)=\left(a+b|u|^{\gamma}\right)|u|^{\mu} \operatorname{sgn} u,
$$

where $a \geq 0, b>0, \gamma \geq 0$ and $\mu \geq 0$ are such that $a+b=1$.

Theorem 3.1. Let $0 \leq p(t) \leq a<1$ or $1<p(t) \leq a<\infty$. Suppose that $\left(H_{1}\right)-\left(H_{7}\right)$ hold. Then every solution of Eq. (1.1) with $\sigma(t)=t-\sigma_{1}$ either oscillates or tends to zero as $t \rightarrow \infty$.

Proof. Due to Remark 3.1, we have $b(t) \rightarrow 0$ as $t \rightarrow \infty$. So $\left(H_{7}\right)$ implies that

$$
\int_{t_{0}}^{\infty} Q(t) \sum_{i=1}^{\ell} G\left(R\left(\tau_{i}(t)\right)\right) d t=\infty .
$$

We assume that $x(t)$ is a nonoscillatory solution of (1.1). Then $x(t)>0$ or $x(t)<0$ for $t \geq t_{0}>\rho$. Let $x(t)>0$ for $t \geq t_{0}$. Setting

$$
\begin{gathered}
z(t)=x(t)+p(t) x(\sigma(t)) \\
K(t)=\int_{t}^{\infty} \frac{s-t}{r_{1}(s)} \int_{s}^{\infty} \sum_{i=1}^{\ell}(\theta-s) h_{i}(\theta) H\left(x\left(\rho_{i}(\theta)\right)\right) d \theta d s
\end{gathered}
$$

and

$$
v(t)=z(t)-K(t)=x(t)+p(t) x(\sigma(t))-K(t)
$$

we have

$$
\left(r_{1}(t) v^{\prime \prime}(t)\right)^{\prime \prime}=-\sum_{i=1}^{\ell} q_{i}(t) G\left(x\left(\tau_{i}(t)\right)\right) \leq 0, \quad \not \equiv 0
$$

for $t \geq t_{0}+\sigma_{1}$. Therefore, $v(t), v^{\prime}(t),\left(r_{1}(t) v^{\prime \prime}(t)\right)$, and $\left(r_{1}(t) v^{\prime \prime}(t)\right)^{\prime}$ are monotonic on $\left[t_{1}, \infty\right), t_{1} \geq t_{0}+\sigma_{1}$. In what follows, we have two cases, $v(t)>0$ or $<0$ for $t \geq t_{1}$. Assume that we have the first case. By Lemma 2.1, any of the 
cases (a), (b), (c), and (d) holds. Suppose that any of the cases (a), (b), and (d) holds. By using $\left(H_{3}\right),\left(H_{4}\right)$, and $\left(H_{6}\right)$, Eq. (1.1) can be represented as

$$
\begin{aligned}
& 0=\left(r_{1}(t) v^{\prime \prime}(t)\right)^{\prime \prime}+\sum_{i=1}^{\ell} q_{i}(t) G\left(x\left(\tau_{i}(t)\right)\right)+G(a)\left(r_{1}(\sigma(t)) v^{\prime \prime}(\sigma(t))\right)^{\prime \prime} \\
& +G(a) \sum_{i=1}^{\ell} q_{i}(\sigma(t)) G\left(x\left(\tau_{i}(\sigma(t))\right)\right) \\
& \geq\left(r_{1}(t) v^{\prime \prime}(t)\right)^{\prime \prime}+G(a)\left(r_{1}(\sigma(t)) v^{\prime \prime}(\sigma(t))\right)^{\prime \prime}+\lambda Q(t) \sum_{i=1}^{\ell} G\left(x\left(\tau_{i}(t)\right)+a x\left(\tau_{i}(\sigma(t))\right)\right) \\
& \geq\left(r_{1}(t) v^{\prime \prime}(t)\right)^{\prime \prime}+G(a)\left(r_{1}(\sigma(t)) v^{\prime \prime}(\sigma(t))\right)^{\prime \prime}+\lambda Q(t) \sum_{i=1}^{\ell} G\left(z\left(\tau_{i}(t)\right)\right)
\end{aligned}
$$

for $t \geq t_{2}>t_{1}$, where we have used the fact that $z(t) \leq x(t)+a x(\sigma(t))$. From (3.3), $K(t)>0, K^{\prime}(t)<0$, and thus, $\lim _{t \rightarrow \infty} K(t)$ exists due to $\left(H_{2}\right)$. Also, the inequality $v(t)>0$ for $t \geq t_{1}$ implies that $v(t)>z(t)$ for $t \geq t_{2}$ and, thus, the $t \rightarrow \infty$
last inequality yields

$\left(r_{1}(t) v^{\prime \prime}(t)\right)^{\prime \prime}+G(a)\left(r_{1}(\sigma(t)) v^{\prime \prime}(\sigma(t))\right)^{\prime \prime}+\lambda Q(t) \sum_{i=1}^{\ell} G\left(v\left(\tau_{i}(t)\right)\right) \leq 0$,

for $t \geq t_{2}$, i.e.,

$\left(r_{1}(t) v^{\prime \prime}(t)\right)^{\prime \prime}+G(a)\left(r_{1}(\sigma(t)) v^{\prime \prime}(\sigma(t))\right)^{\prime \prime}+\lambda G\left(k_{1}\right) Q(t) \sum_{i=1}^{\ell} G\left(R\left(\tau_{i}(t)\right)\right) \leq 0$

due to $\left(H_{4}\right)$ and Lemma 2.3, for $t \geq t_{3}>t_{2}$. Integrating this inequality from $t_{3}$ to $\infty$, we get

$$
\lambda G\left(k_{1}\right) \int_{t_{3}}^{\infty} Q(t) \sum_{i=1}^{\ell} G\left(R\left(\tau_{i}(t)\right)\right) d t<\infty
$$

but this contradicts (3.1). Further, we suppose that the case (c) holds. By using Lemmas 2.2 and 2.3, we have

$$
k\left(-r_{1}(t) v^{\prime \prime}(t)\right)^{\prime} t R(t) \leq v(t) \leq k_{2} t
$$

for $t \geq t_{4}>t_{3}$. Hence,

$$
\begin{gathered}
-\left[\left(\left(-r_{1}(t) v^{\prime \prime}(t)\right)^{\prime}\right)^{1-\gamma}\right]^{\prime}=(\gamma-1)\left(\left(-r_{1}(t) v^{\prime \prime}(t)\right)^{\prime}\right)^{-\gamma}\left(-r_{1}(t) v^{\prime \prime}(t)\right)^{\prime \prime} \\
\geq(\gamma-1) L^{\gamma} R^{\gamma}(t) \sum_{i=1}^{\ell} q_{i}(\sigma(t)) G\left(x\left(\tau_{i}(\sigma(t))\right)\right),
\end{gathered}
$$

where $L=\frac{k}{k_{2}}>0$. Therefore, the inequality

$$
\begin{aligned}
& -\left[\left(\left(-r_{1}(t) v^{\prime \prime}(t)\right)^{\prime}\right)^{1-\gamma}\right]^{\prime}-G(a)\left[\left(\left(-r_{1}(\sigma(t)) v^{\prime \prime}(\sigma(t))\right)^{\prime}\right)^{1-\gamma}\right]^{\prime} \\
& \geq(\gamma-1) L^{\gamma}\left[R^{\gamma}(t) \sum_{i=1}^{\ell} q_{i}(t) G\left(x\left(\tau_{i}(t)\right)\right)+G(a) R^{\gamma}(\sigma(t)) \sum_{i=1}^{\ell} q_{i}(\sigma(t)) G\left(x\left(\tau_{i}(\sigma(t))\right)\right)\right] \\
& \geq \lambda(\gamma-1) L^{\gamma} b(t) Q(t) \sum_{i=1}^{\ell} G\left(z\left(\tau_{i}(t)\right)\right) \geq \lambda(\gamma-1) L^{\gamma} b(t) Q(t) \sum_{i=1}^{\ell} G\left(v\left(\tau_{i}(t)\right)\right) \\
& \geq \lambda(\gamma-1) L^{\gamma} G\left(k_{1}\right) b(t) Q(t) \sum_{i=1}^{\ell} G\left(R\left(\tau_{i}(t)\right)\right) \\
& \text { implies that }
\end{aligned}
$$

$$
\lambda(\gamma-1) L^{\gamma} G\left(k_{1}\right) \int_{t_{4}}^{\infty} b(t) Q(t) \sum_{i=1}^{\ell} G\left(R\left(\tau_{i}(t)\right)\right) d t<\infty,
$$

which contradicts $\left(H_{7}\right)$. Therefore, the latter holds. Consequently, the inequality $z(t)<K(t)$, where $K(t)$ is bounded, implies that $x(t)$ is bounded. It follows from Lemma 2.4 that any of the cases (b)-(f) is realized for $t \geq t_{2}>t_{1}$. In the cases (e) and (f) of Lemma 2.4, we get $\lim _{t \rightarrow \infty} v(t)=-\infty$, which contradicts the facts that $x(t)$ is bounded and $\lim _{t \rightarrow \infty} v(t)$ exists. Keep in view either the case (b) or the case (c), where $-\infty<\lim _{t \rightarrow \infty} v(t) \leq 0$. Hereby, 


$$
\begin{gathered}
0 \geq \lim _{t \rightarrow \infty} v(t)=\limsup _{t \rightarrow \infty}[z(t)-K(t)] \geq \limsup _{t \rightarrow \infty}[x(t)-K(t)] \\
\geq \limsup _{t \rightarrow \infty} x(t)-\lim _{t \rightarrow \infty} K(t)=\limsup _{t \rightarrow \infty} x(t)
\end{gathered}
$$

implies that $\lim _{t \rightarrow \infty} x(t)=0$. We may note that $\lim _{t \rightarrow \infty} K(t)=0$. At last, let the case (d) of Lemma2.4 hold. Then $\lim _{t \rightarrow \infty}\left(r_{1}(t) v^{\prime \prime}(t)\right)^{\prime}$ exists. Hence, integrating (3.5) from $t_{2}$ to $\infty$, we obtain

$$
\int_{t_{2}}^{\infty} \sum_{i=1}^{\ell} q_{i}(t) G\left(x\left(\tau_{i}(t)\right)\right) d t<\infty
$$

i.e.,

$$
\int_{t_{2}}^{\infty} Q(t) \sum_{i=1}^{\ell} G\left(x\left(\tau_{i}(t)\right)\right) d t<\infty .
$$

If $\liminf _{t \rightarrow \infty} x(t)>0$, then inequality (3.7) implies that

$$
\int_{t_{2}}^{\infty} Q(t) d t<\infty,
$$

which contradicts $\left(H_{6}\right)$ due to Remark 3.1. Therefore, $\liminf _{t \rightarrow \infty} x(t)=0$. Since $\lim _{t \rightarrow \infty} v(t)$ exists, by using Lemma 2.5, we get

$$
\lim _{t \rightarrow \infty} v(t)=0=\lim _{t \rightarrow \infty} z(t) .
$$

Even, $z(t) \geq x(t)$ implies that $\lim _{t \rightarrow \infty} x(t)=0$. If $x(t)<0$ for $t \geq t_{0}$, then we set $y(t)=-x(t)$ for $t \geq t_{0}$ and

$$
\left(r_{1}(t)(y(t)+p(t) y(\sigma(t)))^{\prime \prime}\right)^{\prime \prime}+\sum_{i=1}^{\ell} q_{i}(t) G\left(y\left(\tau_{i}(t)\right)\right)-\sum_{i=1}^{\ell} h_{i}(t) H\left(y\left(\rho_{i}(t)\right)\right)=0
$$

Thus Theorem 3.1 is proved.

Remark 3.3. It follows from Theorem 3.1 that $x(t)$ is bounded in the case where $v(t)<0$ for $t \geq t_{1}$, which further converges to zero as $t \rightarrow \infty$. However, this fact is not required in the other case. Hence, the following theorem has been proved.

Theorem 3.2. Let $0 \leq p(t) \leq a<\infty$. Suppose that $\left(H_{1}\right)-\left(H_{7}\right)$ hold. Then every unbounded solution of (1.1) oscillates.

Theorem 3.3. Let $0 \leq p(t) \leq a<1$. If $\left(H_{1}\right),\left(H_{2}\right),\left(H_{4}\right),\left(H_{5}\right)$, and $\left(H_{8}\right)$ hold, then every unbounded solution of (1.1) oscillates.

Proof. Since $R(t) \rightarrow 0$ as $t \rightarrow \infty$. $\left(H_{8}\right)$ implies that

$$
\int_{t_{0}}^{\infty} \sum_{i=1}^{\ell} G\left(R\left(\tau_{i}(t)\right)\right) q_{i}(t) d t<\infty
$$

and, hence,

$$
\int_{t_{0}}^{\infty} \sum_{i=1}^{\ell} q_{i}(t) d t<\infty .
$$

Let $x(t)$ be a nonoscillatory solution of (1.1) such that $x(t)$ is unbounded and $x(t)>0$ for $t \geq t_{0}>0$. The case $x(t)<0$ for $t \geq t_{0}>0$ is similar. We set $z(t), K(t)$, and $v(t)$ as in (3.2), (3.3), and (3.4), respectively, to obtain (3.5) for $t \geq t_{0}+\sigma_{1}$. Consequently, each of $v(t), v^{\prime}(t),\left(r_{1}(t) v^{\prime \prime}(t)\right)$, and $\left(r_{1}(t) v^{\prime \prime}(t)\right)^{\prime}$ is of constant sign on $\left[t_{1}, \infty\right), t \geq t_{0}+\sigma_{1}$. Assume that $v(t)>0$ for $t \geq t_{1}$. Then Lemma 2.1 holds. If any of the cases (a) or (b) holds, then 


$$
0<v^{\prime}(t)=z^{\prime}(t)-K^{\prime}(t)
$$

implies that $z^{\prime}(t)>0$ or $<0$ for $t \geq t_{1}$. Pay attention to $z(t)$ is unbounded because $x(t)$ is unbounded. Thus, $z^{\prime}(t)<0$ is not true. Ultimately, $z^{\prime}(t)>0$ and we obtain

$$
(1-p(t)) z(t)<z(t)-p(t) z(\sigma(t))=x(t)-p(t) p(\sigma(t)) x(\sigma(\sigma(t)))<x(t) .
$$

This means that

$$
x(t)>(1-a) z(t)>(1-a) v(t)
$$

for $t \geq t_{2}>t_{1}$. Hence, (3.5) yields

$$
G\left((1-a) v\left(\tau_{i}(t)\right) q_{i}(t) \leq-\left(r_{1}(t) v^{\prime \prime}(t)\right)^{\prime \prime},\right.
$$

i.e.,

$$
G\left(k_{1}(1-a)\right) G\left(R\left(\tau_{i}(t)\right)\right) q_{i}(t) \leq-\left(r_{1}(t) v^{\prime \prime}(t)\right)^{\prime \prime}
$$

due to Lemma 2.3 and $\left(H_{4}\right)$. Integrating (3.10) from $t_{2}$ to $\infty$, we conclude that

$$
\int_{t_{2}}^{\infty} \sum_{i=1}^{\ell} G\left(R\left(\tau_{i}(t)\right)\right) q_{i}(t) d t<\infty
$$

which contradicts (3.8). For the case (c) of Lemma 2.1, we proceed as in the proof of Theorem 3.1 to obtain (3.6). By using inequality (3.6), we obtain

$$
-\left[\left(\left(-r_{1}(t) v^{\prime \prime}(t)\right)^{\prime}\right)^{1-\gamma}\right]^{\prime} \geq(\gamma-1) L^{\gamma} G\left((1-a) k_{1}\right) R^{\gamma}(t) \sum_{i=1}^{\ell} q_{i}(t) G\left(R\left(\tau_{i}(t)\right)\right)
$$

for $t \geq t_{2}$. Integrating the last inequality from $t_{2}$ to $\infty$, we find

$$
\int_{t_{2}}^{\infty} R^{\gamma}(t) \sum_{i=1}^{\ell} q_{i}(t) G\left(R\left(\tau_{i}(t)\right)\right) d t<\infty
$$

which contradicts $\left(H_{8}\right)$. In the case $(\mathrm{d})$ of Lemma 2.1, $\lim _{t \rightarrow \infty} v(t)$ exists, i.e., $\lim _{t \rightarrow \infty} z(t)$ exists in contradiction with our hypothesis. Due to Remark 3.3, the case $v(t)<0$ is not executed. Thus, Theorem 3.3 is proved.

Theorem 3.4. Let $-1<a \leq p(t) \leq 0$. If $\left(H_{1}\right),\left(H_{2}\right),\left(H_{5}\right)$ and $\left(H_{8}\right)$ hold, then every solution of Eq. (1.1) with $\sigma(t)=t-\sigma_{1}$ is either oscillatory or tends to zero as $t \rightarrow \infty$.

Proof. Let $x(t)$ be a nonoscillatory solution of (1.1) such that $x(t)>0$ for $t \geq t_{0}>0$.Setting $z(t), K(t)$, and $v(t)$ as in (3.2), (3.3) and (3.4) we obtain (3.5) for $t \geq t_{0}+\sigma_{1}$ and, therefore, $v(t)$ is monotone on $\left[t_{1}, \infty\right), t_{1} \geq t_{0}+\sigma_{1}$. Let $v(t)>0$ for $t \geq t_{1}$. Assume that one of the cases (a), (b) and (d) of Lemma 2.1 holds for $t \geq t_{1}$. From Lemma 2.3, we conclude that $x(t) \geq v(t) \geq k_{1} R(t)$ for $t \geq t_{2}>t_{1}$ and, hence, (3.5) yields

$$
\int_{t_{3}}^{\infty} \sum_{i=1}^{\ell} q_{i}(t) G\left(R\left(\tau_{i}(t)\right)\right) d t<\infty, \quad t_{3}>t_{2}+\sigma_{1}
$$

which contradicts (3.8). Now consider the case (c). Proceeding as in the proof of Theorem 3.1 we have (3.6). Further, $x(t) \geq v(t) \geq k_{1} R(t)$ for $t \geq t_{2}$ by Lemma 2.3. Consequently, for $t \geq t_{3}>t_{2}+\alpha$,

$$
-\left[\left(\left(-r_{1}(t) v^{\prime \prime}(t)\right)^{\prime}\right)^{1-\gamma}\right]^{\prime} \geq(\gamma-1) L^{\gamma} G\left(k_{1}\right) R^{\gamma}(t) \sum_{i=1}^{\ell} q_{i}(t) G\left(R\left(\tau_{i}(t)\right)\right) .
$$

Integrating above inequality from $t_{3}$ to $\infty$, we obtain 


$$
\int_{t_{3}}^{\infty} R^{\gamma}(t) \sum_{i=1}^{\ell} q_{i}(t) G\left(R\left(\tau_{i}(t)\right)\right) d t<\infty
$$

in contradiction with $\left(H_{8}\right)$.

If $v(t)<0$ for $t \geq t_{1}$, then $x(t)$ is ultimately bounded. Thus, $z(t)$ is bounded and the same is true for $v(t)$. In what follows, none of the cases (e) and (f) of Lemma 2.4 is executed. In the case (b)[or (c)], we have

$$
-\infty<\lim _{t \rightarrow \infty} v(t) \leq 0 .
$$

In view of the fact that $\lim _{t \rightarrow \infty} K(t)=0$, we obtain

$$
\lim _{t \rightarrow \infty} v(t)=\lim _{t \rightarrow \infty} z(t)
$$

Hence,

$0 \geq \lim _{t \rightarrow \infty} v(t)=\lim _{t \rightarrow \infty} z(t)=\limsup _{t \rightarrow \infty}[x(t)+p(t) x(\sigma(t))] \geq \limsup _{t \rightarrow \infty} x(t)+\liminf _{t \rightarrow \infty}(a x(\sigma(t)))$

$$
=\limsup _{t \rightarrow \infty} x(t)+a \limsup _{t \rightarrow \infty} x(\sigma(t))=(1+a) \limsup _{t \rightarrow \infty} x(t)
$$

implies that $\limsup _{t \rightarrow \infty} x(t)=0$, i.e., $\lim _{t \rightarrow \infty} x(t)=0$. Let the case $(\mathrm{d})$ hold. Since

$$
\lim _{t \rightarrow \infty}\left(r_{1}(t) v^{\prime \prime}(t)\right)^{\prime}
$$

exists, (3.5) implies that

$$
\int_{t_{2}}^{\infty} \sum_{i=1}^{\ell} q_{i}(t) G\left(x\left(\tau_{i}(t)\right)\right) d t<\infty .
$$

If $\liminf _{t \rightarrow \infty} x(t)>0$, then it follows from (3.11) that

$$
\int_{t_{2}}^{\infty} \sum_{i=1}^{\ell} q_{i}(t) d t<\infty
$$

which contradicts (3.9). Therefore, $\liminf _{t \rightarrow \infty} x(t)=0$. In view of Lemma 2.5, we assert that

$$
\lim _{t \rightarrow \infty} v(t)=0=\lim _{t \rightarrow \infty} z(t) .
$$

Following the above proof, we can see that $\limsup _{t \rightarrow \infty} x(t)=0$ and, hence, $\lim _{t \rightarrow \infty} x(t)=0$.

If $x(t)<0$ for $t \geq t_{0}$, then, acting as above, we obtain $\liminf _{t \rightarrow \infty} x(t)=0$. This means that $\lim _{t \rightarrow \infty} x(t)=0$.

Thus, Theorem 3.4 is proved.

Theorem 3.5. Let $-\infty<p(t) \leq 0$. If $\left(H_{1}\right),\left(H_{2}\right),\left(H_{5}\right)$ and $\left(H_{8}\right)$ hold, then every unbounded solution of (1.1) with $\sigma(t)=t-\sigma_{1}$ is oscillatory.

The proof of this theorem is quite similar the proof of Theorem 3.4. Hence, the details are omitted. 


\section{References}

[1] Tripathy, A. K., Oscillation properties of a class of neutral differential equations with positive and negative coefficients. Fasc. Math. 45 (2010), 133-155.

[2] Tripathy, A. K., Panigrahi, S. and Basu, R., Oscillation results for fourth-order nonlinear neutral differential equations with positive and negative coefficients. Journal of Mathematical Sciences 194 (2013), no. 4, 453-471.

[3] Gyori, I. and Ladas, G., Oscillation theory of delay differential equation with application. Clarendon Press. Oxford, 1991.

[4] Parhi, N. and Tripathy, A. K., On oscillatory fourth-order nonlinear neutral differential equations. I, Math. Slovaca 54 (2004), 389-410.

[5] Parhi, N. and Chand, S., On forced first-order neutral differential equations with positive and negative coefficients. Math. Slovaca 50 (2000), 183-202.

[6] Ocalan, O., Oscillation of forced neutral differential equations with positive and negative coefficients. Comput. Math. and Appl. 54 (2007), 1411-1421.

[7] Ocalan, O., Oscillation of neutral differential equation with positive and negative coefficients. J. Math. Anal. and Appl. 331 (2007), 644-654.

[8] Chuanxi, Q. and Ladas, G., Oscillation in differential equations with positive and negative coefficients. Can. Math. Bull. 33 (1990), 442-450.

[9] Edwards, R. E., Functional analysis. Holt, Rinehart and Winston Inc. New York, 1965.

[10] Li, W. T. and Quan, H. S., Oscillation of higher order neutral differential equations with positive and negative coefficients. Ann. Different. Equat. 2 (1995), 70-76.

[11] Li, W. T. and Yan, J., Oscillation of first-order neutral differential equations with positive and negative coefficients. Collect. Math. 50 (1999), 199-209.

\section{Affiliations}

FIRST AUTHOR

ADDRESS: Erciyes University, Dept. of Mathematics, Faculty of Science, Kayseri-TURKEY.

E-MAIL: temtek@erciyes.edu.tr

SECOND AUTHOR

ADDRESS: Ahi Evran University, Dept. of Mathematics, Faculty of Science and Arts, Kırşehir-TURKEY.

E-MAIL: nagehan.kilinc@ahievran.edu.tr 